\title{
Tunnelmagnetoresistive Sensoren für Weg-, Winkel- und Positionsmessung in der elektrischen Antriebstechnik
}

\author{
Dr. Rolf Slatter \\ Sensitec GmbH, Lahnau, Deutschland
}

\section{Zusammenfassung}

Längen- und Winkelmesssysteme auf magnetischer Basis erleben in den letzten Jahren ein rasantes Wachstum. Allen voran finden Sensoren auf Basis des magnetoresistiven (MR) Effekts zunehmend Anwendung in der Antriebstechnik, sowohl im industriellen als auch im automotiven Bereich. MRSensoren werden nicht nur bei der Regelung von mechatronischen, sondern auch bei pneumatischen und hydraulischen Antrieben eingesetzt. Es können inkrementelle als auch absolute Messungen in einer Vielzahl von unterschiedlichen Konfigurationen durchgeführt werden. Neue Sensoren auf Basis des tunnelmagnetoresistiven (TMR) Effektes eröffnen neue Möglichkeiten aufgrund ihrer sehr geringen Abmessungen, ihrem exzellenten Temperaturverhalten und dem niedrigen Energiebedarf. Damit bilden sie eine wichtige Ergänzung für die bisher eingesetzten AMR- und GMR-Sensoren in antriebstechnischen Anwendungen.

Keywords: Magnetische Sensoren, Winkelmessung, Wegmessung, Positionsmessung, magnetoresistive Sensoren

\section{Einleitung}

Der Bedarf an mechatronischen Antrieben steigt in mehreren Industriebereichen. Nicht nur bei der Industrieautomatisierung, sondern auch in der Fahrzeugtechnik und der Luftfahrt nimmt die Anzahl der eingesetzten Antriebe stetig zu. „Dezentralisierte Antriebstechnik“, "elektrifizierte Antriebstrang“ oder „more electric aircraft" sind nur einige der Begriffe, die diesen Trend in den verschiedenen technologischen Bereichen beschreiben. Diese Entwicklung stellt neue Anforderungen an die eingesetzte Sensortechnologie für die Messung von linearen und rotatorischen Bewegungen. Abb. 1 zeigt die Schematik eines elektrischen Antriebes, typischerweise bestehend aus einem Umrichter, Servomotor, Drehzahlund Positionssensoren (Encoder) sowie mechanischer Maschinenelemente. Aktuell entstehen neue Bedürfnisse, die die technischen und wirtschaftlichen Anforderungen, die sowohl an Antriebe als auch an Sensoren gestellt werden, neu definieren. Die hohe Leistung und Flexibilität von magnetoresistiven (MR) Sensoren spielt eine zunehmend wichtige Rolle bei der Unterstützung von Maschinen- und Antriebsentwicklern, um diesen neuen Bedürfnissen gerecht zu werden. Diese kompakten, präzisen und robusten Sensoren bieten Eigenschaften, die von optischen, kapazitativen oder induktiven Sensoren nicht geboten werden.

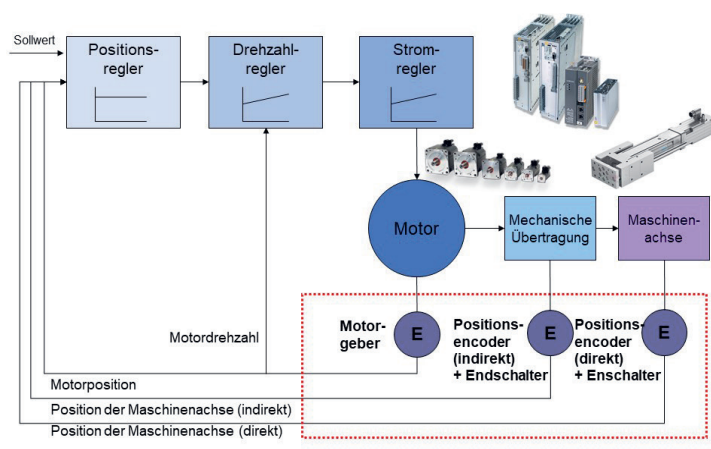

Abb. 1: Schematik eines elekromechanischen Antriebs (Bildquellen: LTi Motion GmbH, Festo $A G)$

\section{Entwicklungstrends in der elektrischen Antriebstechnik}

Der Markt für Antriebstechnik wird durch mehrere aktuelle Trends bestimmt, die neue Anforderungen sowohl an Antriebe als auch Sensoren stellen:

\section{a) Höhere Genauigkeit}

Aus der zunehmenden Bedeutung von Mikrosystemtechnik sowie Nanotechnologie ergeben sich neue Ziele bezüglich der Genauigkeit von Robotern, Werkzeugmaschinen und anderen Formen der Industrieautomatisierung. Es steigen nicht nur die Anforderungen hinsichtlich Positioniergenauigkeit, sondern auch die An- 
forderungen an eine höhere Auflösung zwecks höherer Bahngenauigkeit. Hinzu kommt, dass die gewünschte Genauigkeit über die Lebensdauer der Maschine unter unterschiedlichen Betriebsbedingungen konstant bleiben soll. Dies führt wiederum zum verstärkten Einsatz von Direktmesssystemen.

b) Höhere Leistungsdichte

Maschinen erhalten zunehmend kompaktere Bauformen, um den verfügbaren Bauraum optimal zu nutzen und auch um die Energieeffizienz zu steigern. Dadurch steigt die Leistungsdichte von Antrieben stetig an, was sich in höheren Abtriebsdrehmomenten sowie höheren Geschwindigkeiten ohne einen proportionalen Anstieg der physikalischen Größe niederschlägt.

\section{c) Dezentralisierte Antriebstechnik}

Die verbesserte Rechenkapazität von MikroControllern führt zu einer Verlagerung von Mess- und Regelungsaufgaben bis auf die Ebene des einzelnen Antriebs herunter. Dezentralisierte Antriebe, bestehend aus Servomotoren mit integrierter Elektronik, bedürfen weniger Verkabelung, geringeren Montageaufwand und entlasten höher geordnete Steuerungen. Damit lassen sich die Kosten von Maschinen, die über viele Antriebe verfügen, drastisch senken.

d) Zunehmender Einsatz von geschlossenen Regelkreisen

Elektromechanische Antriebe werden zunehmend in Anwendungen eingesetzt, die früher ausschließlich durch hydraulische oder pneumatische Antriebe besetzt waren. Um diesem Trend entgegenzuwirken, setzen die Hersteller der letztgenannten immer häufiger geschlossenen Regelkreise ein, um die Genauigkeit und Dynamik ihrer Antriebe zu steigern.

e) Immer anspruchsvollere Umgebungsbedingungen

Immer mehr Anwendungen finden unter sehr niedrigen oder sehr hohen Umgebungstemperaturen statt. Es gibt bekannte Beispiele in der Luft- und Raumfahrt, aber auch im industriellen Bereich steigt die Zahl der Anwendungen, die unter extremen Temperatur- und Umgebungsbedingungen funktionieren müssen.

\section{f) Energieeffizienz}

Der Anzahl batteriebetriebenen Anwendungen steigt stetig. Sensoren mit sehr niedrigen Energiebedarf ermöglichen eine längere Batterie-Lebensdauer bzw. den Einsatz kleinerer Batterien in mobilen Anwendungen. g) Schnelleres „Time-to-market“

Die Lebenszyklen von nahezu allen Maschinentypen werden verkürzt. Das bedeutet, dass auch die Entwicklungszeiten kürzer werden, um eine schnelle Amortisation des Entwicklungsaufwandes zu gewährleisten. Dies hat zur Folge, dass Maschinen- und Antriebskonstrukteure nach besonders verlässlichen Sensorlösungen mit einem hohen Integrationsgrad suchen.

Diese Trends haben eine unmittelbare Auswirkung auf die Sensoren, die für Längen- und Winkelmessaufgaben eingesetzt werden.

\section{Neue Anforderungen an die Sensorik}

Der Konstrukteur muss ein Messsystem auswählen, das folgende Eigenschaften vereint:

- Hohe Auflösung für hohe Regelgüte

- Hohe Genauigkeit für präzise Positionierung

- Hohe Bandbreite, um den Einsatz auch bei sehr hohen Geschwindigkeiten und Drehzahlen zu ermöglichen

- Einfache, günstige Montage und Einrichtung

- Einsetzbar unter schwierigen Umgebungsbedingungen (hohe oder niedrige Temperaturen, Verschmutzung, hohe mechanische Belastung usw.)

- Unempfindlichkeit gegen Schock und Vibration

- Geringes Bauvolumen

- Hohe Energieeffizienz

- Hohe Zuverlässigkeit

Diese komplexe Anforderungsliste kann durch Messsysteme, die auf magnetoresistiven Sensoren basieren, vollständig erfüllt werden. Lineare und Dreh-Geber auf MR-Basis bieten mehrere Vorteile gegenüber anderen Messprinzipien. Optische Geber verfügen über eine hohe Genauigkeit, bieten aber auf Grund begrenzter Linearität eine niedrigere Auflösung. Die höchste zulässige Einsatztemperatur für optische Geber liegt oft nur bei $85^{\circ} \mathrm{C}$ und nur selten bei $100{ }^{\circ} \mathrm{C}$. MR-Sensoren können bei deutlich höheren Temperaturen eingesetzt werden und sind bis $150^{\circ} \mathrm{C}$ qualifiziert, sowohl für Industrie- als auch Automobilanwendungen. Optische Geber bieten zudem ein begrenztes Miniaturisierungspotenzial auf Grund des komplexen Aufbaus. Ein entscheidender Vorteil von magnetischen Gebern ist die deutlich höhere Robustheit und Widerstandsfähigkeit gegenüber Verschmutzung. Diese Vorteile bieten die Grundlage für den stetig steigenden Marktanteil magnetischer Messsysteme. 


\section{Grundlagen der tunnelmagnetoresisti- ven Sensortechnologie}

Der magnetische Tunnelwiderstand ist ein magnetoresistiver Effekt, der in magnetischen Tunnelkontakten auftritt. In seiner einfachsten Form handelt es sich um ein Bauelement bestehend aus zwei Ferromagneten (Free Layer und Pinned Layer, dargestellt in Abb. 2), die durch einen dünnen Isolator getrennt sind. Ist die isolierende Schicht oder Tunnelbarriere (Barrier Layer, dargestellt in Abb. 2) extrem dünn (einige wenige Nanometer, ähnlich groß wie der Durchmesser der menschlichen DNA), so können Elektronen zwischen den beiden Ferromagneten „tunneln“. Dieser Vorgang ist mit Hilfe der klassischen Physik nicht erklärbar und ist daher ein rein quantenmechanisches Phänomen [1].

Unter dem Einfluss eines äußeren Magnetfeldes kann die Richtung der Magnetisierung der beiden magnetischen Schichten unabhängig voneinander gesteuert werden. Wenn die Magnetisierungen gleich ausgerichtet sind, ist die Wahrscheinlichkeit, dass Elektronen durch die Isolatorschicht hindurch tunneln größer als bei gegensätzlicher Ausrichtung. Damit kann der elektrische Widerstand zwischen unterschiedlichen Widerstandszuständen ( $\Delta R / R)$ variieren.

Obwohl der TMR-Effekt seit den 1970er Jahren bekannt war, wurde es erst durch verbesserte Prozesstechnik ab Mitte der 1990er Jahre möglich, reproduzierbare Ergebnisse zu erzielen. Seit wenigen Jahren hat die Entwicklung nun auch in die Industrie Einzug gehalten, so dass inzwischen Festplatten mit TMR- statt GMR- (Giant MR-Effekt) Schreib-Leseköpfen angeboten werden. Trotzdem ist auf dem Gebiet der Sensorik bisher vornehmlich mit AMR (Anisotroper MR-Effekt) und GMR gearbeitet worden (Abb. 2). Die TMR-Sensoren werden auch in Dünnschichttechnologie hergestellt, wobei wesentliche Teile der Produktionskette für GMR-Sensoren übernommen werden können.
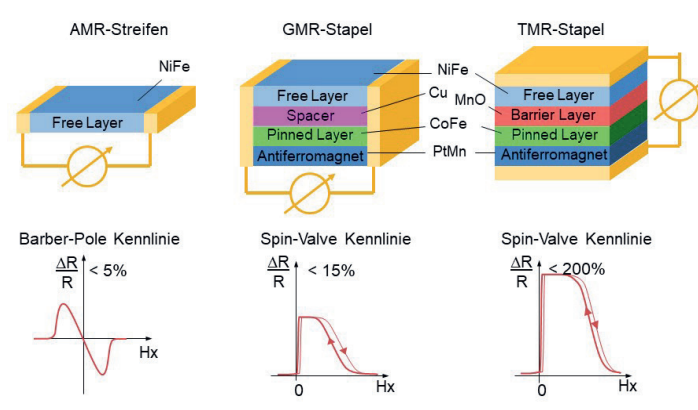

Abb. 2: Vergleich der xMR-Technologien
Der Widerstand des TMR-Sensors ist definiert durch die Fläche der Tunnelverbindung und die Schichtdicke bzw. den Flächenwiderstand der Barriere. Ein typischer Flächenwiderstand einer $\mathrm{Al}_{2} \mathrm{O}_{3}$ Barriere liegt bei $10 \mathrm{M} \Omega / \mu^{2}$. Gestaltet man eine Tunnelbarriere von $100 \mu \mathrm{m}^{2}$ Fläche, so ergibt sich ein Widerstand von 100 $\mathrm{k} \Omega$ aus einem einzigen Tunnelelement.

Im Gegensatz zu AMR- und GMR-Sensoren gilt: je kleiner das Sensorelement, desto größer der Widerstand. Kleinere Tunnelelemente führen zu einem höheren Widerstand. Dies ist ein elementarer Unterschied zu herkömmlichen AMR- und GMR-Sensoren. Er ermöglicht auf gleichem Platz wesentlich hochohmigere Sensoren zu platzieren als es mit AMR- oder GMR-Sensoren möglich ist. Dadurch kann mit TMR der Leistungsverbrauch um einen Faktor 100 bis 1000 reduziert werden [2, 3, 4].
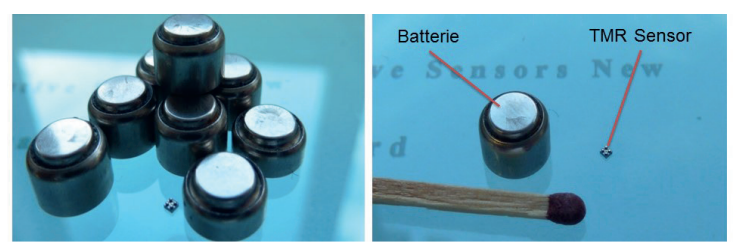

Abb. 3: Bedeutung des Energieverbrauchs in der Sensorik: Deutlich reduzierter Energiebedarf durch TMR-Sensor (rechts) im Vergleich mit AMR-Sensor (links) in einer beispielhaften Anwendung

\section{Anwendungsbeispiele}

5.1 Winkelsensorik

Ein weiterer ganz wesentlicher Unterschied zwischen AMR-Sensoren und GMR- bzw. TMR-Sensoren ist das Verhalten im Drehfeld. Dreht bei einem AMR-Sensor die Magnetisierung um $90^{\circ}$ von parallel zur Stromrichtung auf senkrecht zur Stromrichtung, ist bereits der komplette MR-Hub erschöpft und bei $180^{\circ}$ ist der Ausgangszustand wiedergegeben. Die Periodizität ist demnach nur $180^{\circ}$. Im Gegensatz dazu ist bei GMR- und TMR-Systemen der Ausgangszustand erst nach einer vollen Umdrehung wieder erreicht und somit eine Periodizität von $360^{\circ}$ gegeben $[5,6]$.

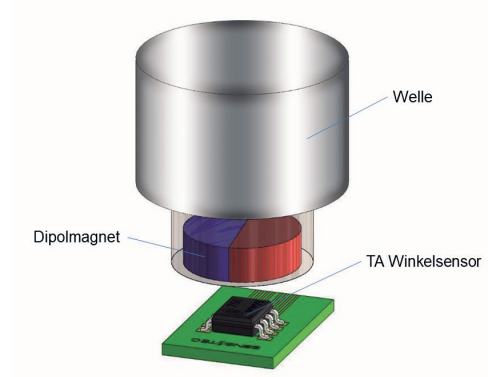

Abb. 4: Typische Messanordnung 


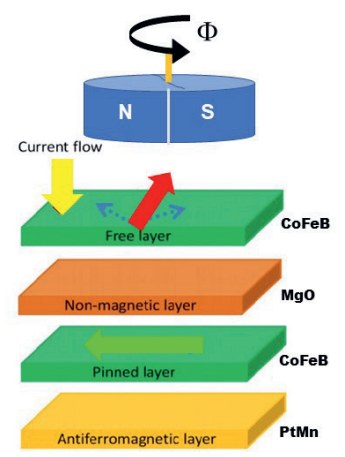

Abb. 5: Funktionsprinzip eines TMR-Winkelsensors

TMR Sensoren sind damit sehr gut geeignet für Anwendungen in der Winkelsensorik, z. B. in Drehgebern am Wellenende eines Antriebs. Eine typische Messanordnung sowie das Funktionsprinzip sind in Abb. 4 bzw. Abb. 5 dargestellt. Üblicherweise wird ein Dipolmagnet an der drehenden Welle angebracht. Eine Drehbewegung der Antriebswelle resultiert in einer Änderung in der Magnetisierungsrichtung des Free Layers, was wiederum zu einer Widerstandsänderung führt.

Um höchste Anforderungen an Signalqualität zu erfüllen, werden die MR-Streifen im PerfectWave ${ }^{\circledR}$ Design ausgeführt. Die Streifen haben eine gekrümmte Form (Abb. 6), die zur Oberwellenfilterung bei der Abbildung der Magnetfeldrichtung in ein elektrisches Signal genutzt wird. Diese Filterung wird durch die spezielle Geometrie und Anordnung der TMRStrukturen realisiert und verursacht keine zusätzlichen Signallaufzeiten. PerfectWave ${ }^{\circledR}$ wirkt sich besonders bei kleineren Magnetfeldern in verbesserter Linearität, höheren Genauigkeiten und dadurch besserer Regelgüte aus. Durch die Oberwellenfilterung werden mehrere Oberwellen unterdrückt, und das analoge Sensorausgangssignal gleicht dadurch nahezu einem perfekten Sinus.

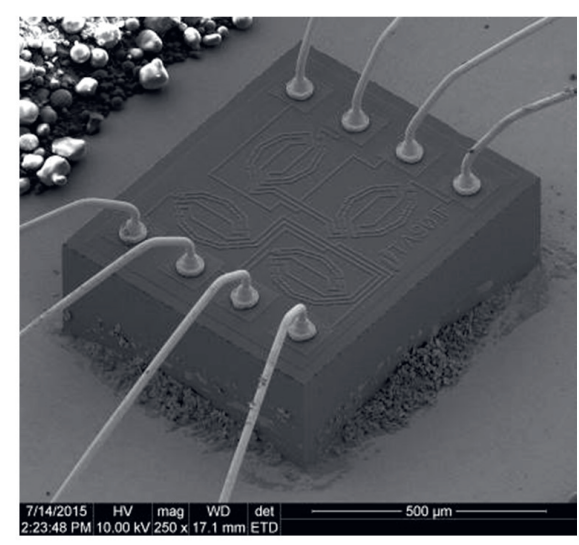

Abb. 6: REM-Aufnahme eines TMRWinkelsensor-Chips
Durch dieses Verfahren können absolute Winkelgenauigkeiten von besser als $0,4^{\circ}$ bei Raumtemperatur und spezifizierte Einbautoleranzen über einen breiten Feldstärkenbereich erreicht werden (Abb. 7) [7].

Bei dem quantitativen Vergleich von Leistungseigenschaften von gängigen AMR- und TMR-Winkelsensoren, dargestellt in Tabelle 1, erkennt man, dass der AMR Sensor weiterhin eine höhere Genauigkeit und niedrigere Hysterese aufweist. Der TMR-Sensor kann jedoch eine absolute Winkelmessung über $360^{\circ}$ ermöglichen und weist eine exzellente Temperaturstabilität aus, was in vielen Anwendungen einen Temperaturabgleich überflüssig macht. Weiterhin bedeutet die hohe Signalamplitude, dass oft auf eine Verstärkung des AnalogSensorsignals verzichtet werden kann, was wiederum zu Kostenersparnissen führen kann.

Tab. 1: Vergleich AMR- und TMRWinkelsensoren

\begin{tabular}{|l|l|l|}
\hline & \multicolumn{1}{|c|}{ AMR } & \multicolumn{1}{c|}{ TMR } \\
\hline Periodizität & $180^{\circ}$ & $360^{\circ}$ \\
\hline Genauigkeit & $0.1^{\circ}$ & $0.4^{\circ}$ \\
\hline Signal Amplitude & $12 \mathrm{mV} / \mathrm{V}$ & $80 \mathrm{mV} / \mathrm{V}$ \\
\hline Amplitude TK & $-0,35 \% / \mathrm{K}$ & $-0,15 \% / \mathrm{K}$ \\
\hline Offset & $2 \mathrm{mV} / \mathrm{V}$ & $3 \mathrm{mV} / \mathrm{V}$ \\
\hline Relativer Offset TK & $\sim 1,6 \%$ @100 K & $\sim 1 \%$ @100 K \\
\hline Widerstand TK & $0,42 \% / \mathrm{K}$ & $-0,1 \% / \mathrm{K}$ \\
\hline Hysterese & vernachlässigbar & $0,1 \%$ \\
\hline Sensorwiderstand & Meanderfläche & Barrieredicke \\
\hline
\end{tabular}

a)

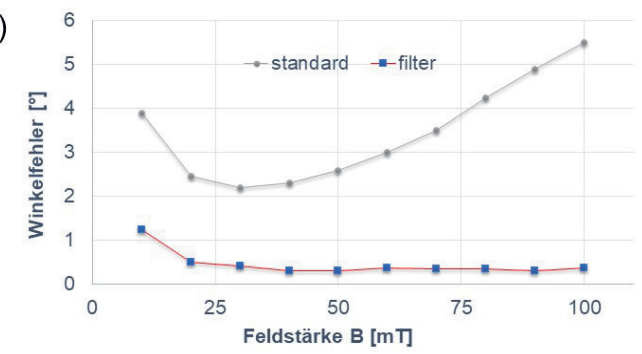

b)

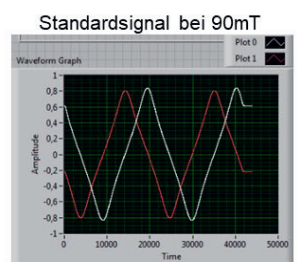

Gefilterte Signal bei $90 \mathrm{mT}$

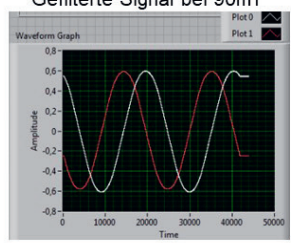

Abb. 7: Oberwellenfilterung 
TMR-Sensoren sind nicht nur für die Winkelmessung am Wellenende anwendbar, sondern auch am Wellenumfang. Um letzteres zu ermöglichen, wird das Nonius-Prinzip angewandt, d. h. es werden zwei oder drei Spuren mit unterschiedlichen Polteilungen benutzt und der Winkel wird durch die unterschiedlichen Phasenlagen der Signale der jeweiligen Sensoren (ein Sensor pro Spur) berechnet. In Zusammenarbeit mit NTN-SNR hat Sensitec eine neue Lösung entwickelt mit einer Auflösung bis 17 bit/Umdrehung (131.072 Puls/Umdrehung) sowie einer sehr hohen absoluten Winkelgenauigkeit von $\pm 0,1^{\circ}(\mathrm{Abb} .8)$.

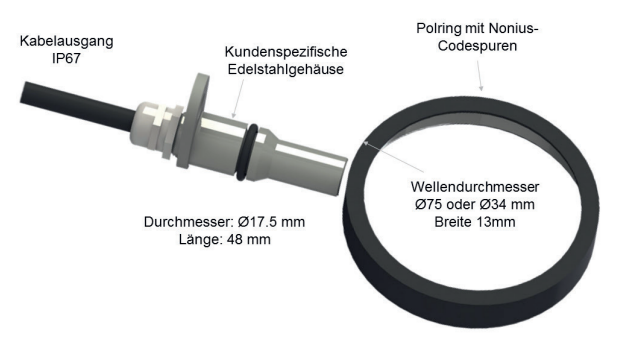

Abb. 8: Absolut-Winkelmesssystem auf TMRBasis (Bildquelle: NTN-SNR Bearings $\mathrm{GmbH}$ )

\subsection{Längensensorik}

Das Nonius-Prinzip kann auch bei AbsolutLängenmesssystemen angewandt werden (Abb. 9). Auch hier verfügt der magnetische Maßstab über zwei oder drei Spuren mit unterschiedlichen Polteilungen. Alternativ verfügt der Maßstab über eine inkrementelle CodeSpur in Verbindung mit einer Spur mit einer Pseudo-Random-Codierung.

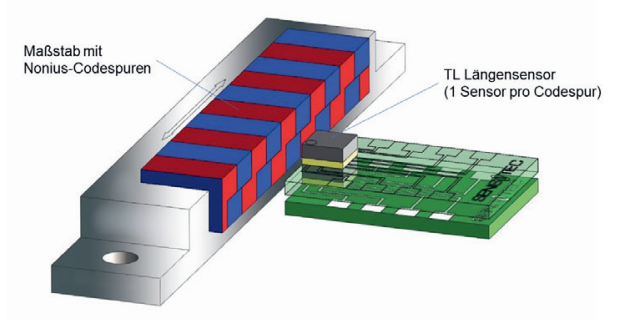

Abb. 9: Absolute Wegmessung mittels NoniusPrinzip

Für solche Anwendungen kommen Längensensoren auf TMR-Basis zum Einsatz. Diese Sensoren sind an der Polteilung des Maßstabes angepasst und verfügen weiterhin über eine Oberwellenfilterung. Sie weisen eine extrem konstante Signalamplitude und absolute Genauigkeit über einer breiten Arbeitsabstandsbereich auf (Abb. 9 bzw. Abb.10).

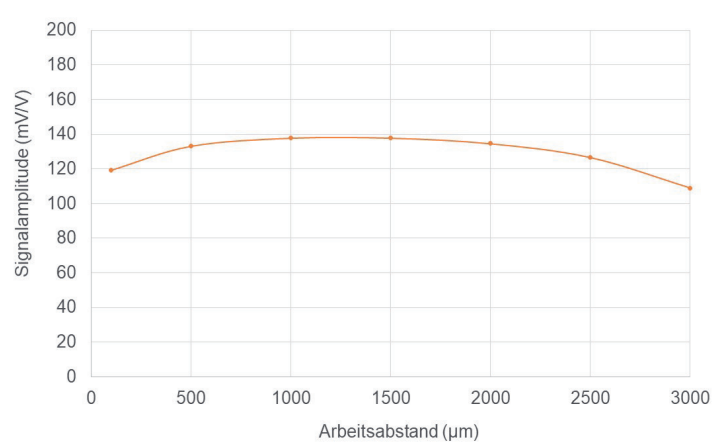

Abb. 10: Signalamplitude vs. Arbeitsabstand

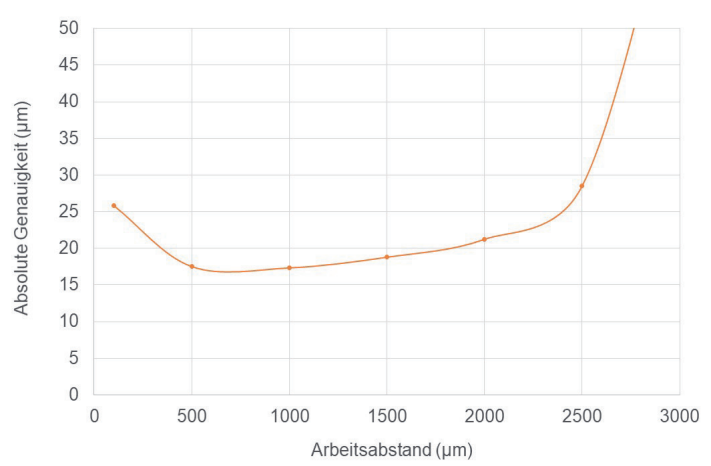

Abb. 11: Absolute Genauigkeit vs. Arbeitsabstand

Innerhalb des BMBF-geförderten Forschungsprojekts KaLiPso - Kabellose Linearführungssysteme mit integrierter Positionsmessung - ist es gelungen, durch die Zusammenarbeit zwischen NTN-SNR sowie dem Institut für Fertigungstechnik und Werkzeugmaschinen an der Universität Hannover als auch Sensitec, ein kabelloses Absolut-Längenmesssystem auf TMR-Basis in einer Linearführung zu integrieren (Abb 12). Wie in Abb. 13 gezeigt ist das Längenmesssystem in der Linearführung integriert und wird mittels eines berührungslosen induktiven Verfahrens mit Energie versorgt. Die Primärspule ist in die Führungsschiene integriert und die Sekundärspule befindet sich im Schlitten [8].

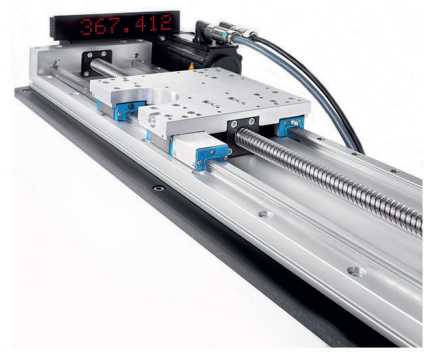

Abb. 12: Linearachse (Bildquelle: NTN-SNR Bearings $\mathrm{GmbH}$ ) 


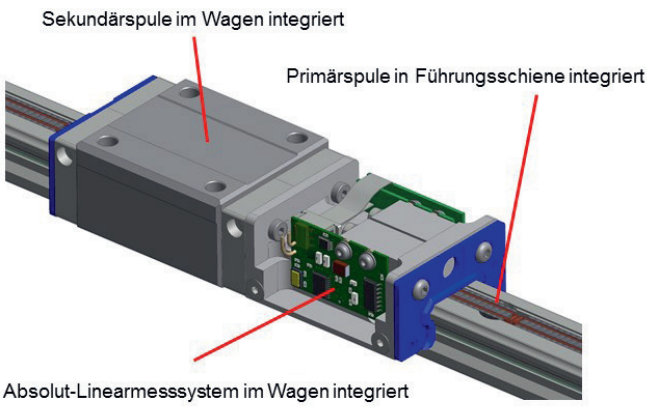

Abb. 13: Integriertes Absolut-Linearmesssystem auf TMR-Basis (Bildquelle: NTN-SNR Bearings $\mathrm{GmbH}$ )

Es gibt gleichzeitig einen Bedarf an Längenmesssystemen für kurze Hübe mit sehr kompakten Abmessungen für die Integration in z. B. Miniatur-Linearführungen oder sehr kleine Wälzlager (Abb. 14). Im kürzlich abgeschlossenen BMBF-geförderten Projekt „Aquila“ wurden lithographisch hergestellte Maßverkörperungen entwickelt, um absolute Positioniergenauigkeiten von $<1 \mu \mathrm{m}$ zu erreichen [9].

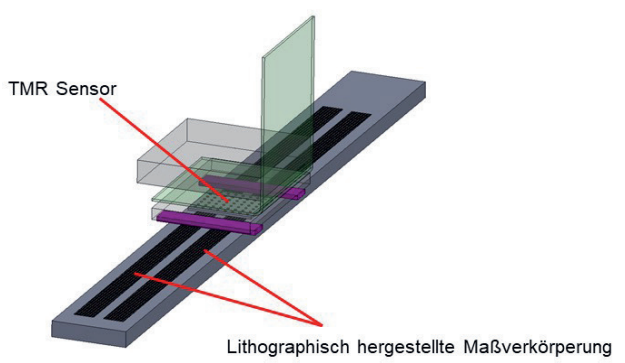

Abb. 14: Grundkonzept des miniaturisierten Wegmesssystems

Der magnetische Maßstab verfügt über zwei hartmagnetische Code-Spuren (eine inkrementelle, eine absolute), die mittels lithographischer Verfahren auf einem Silizium-Substrat strukturiert werden (Abb. 15).
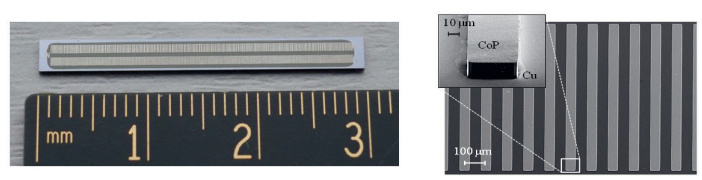

Abb. 15: Lithographisch hergestellte magnetische Maßstäbe

Die Polteilung beträgt nur $20 \mu \mathrm{m}$, eine Größenordnung feiner als bei bisherigen magnetischen Maßstäben, bei denen die kleinsten Polteilungen üblicherweise zwischen 200 und $500 \mu \mathrm{m}$ lagen. Ein speziell entwickelter TMRLängensensor liest beide Code-Spuren gleichzeitig, um eine Absolut-Längenmessung zu realisieren (Abb. 16, 17).
Die extrem kleine Polteilung bedeutet, dass der Luftspalt zwischen Sensor und Maßstab so klein ist, dass konventionelle Wirebonds zwischen Sensorchip und Leiterplatte nicht anwendbar sind. Daher mussten neue Aufbauund Verbindungstechniken untersucht werden, um die empfindliche Fläche des Sensorchips so nah wie möglich an die Maßverkörperung zu bringen.

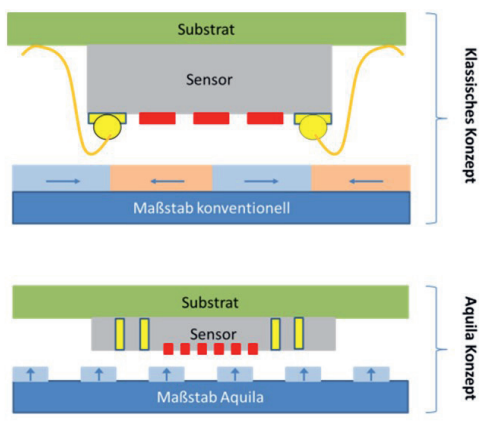

Abb. 16: TMR Längensensor mit „ThroughSilicon-Vias" (TSV)

Through-silicon vias (TSV) sind vertikale elektrische Verbindungen, die durch den Sensorchip geführt werden und eine Kontaktierung auf der Rückseite des Chips erlauben. Die TSVs ermöglichen zudem eine deutliche Reduzierung der Chipfläche und vermindern damit auch die Gesamtabmessungen des Messsystems [10]. Wie aus Abb. 17 zu entnehmen ist, verfügt der Maßstab über zwei Kodespuren - eine Spur für hochauflösende inkrementelle Weginformation, die zweite Spur mit einer speziellen Kodierung für AbsolutWeginformation (PBRS - Pseudo-RandomKodierung). Der Sensorchip hat zwei empfindliche Bereiche, die an die jeweiligen Kodespuren angepasst sind.

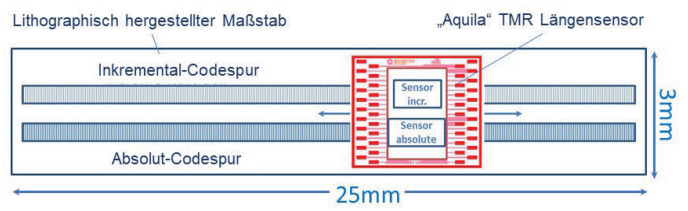

Abb. 17: Prinzip der absoluten Wegmessung

Mit einem Laserinterferometer als Referenzpositionsgeber (Messunsicherheit $<120 \mathrm{~nm} / \mathrm{m}$ ) wurde die Genauigkeit des AQUILA-Systems gemessen. Abb. 18 zeigt oben die Auswertung der Absolutmessung: Das PRBS-Signal ist über den gesamten Maßstab eindeutig. Damit wird die "true-power-on" Ermittlung der Absolutposition nachgewiesen. Direkt nach dem Einschalten kann fehlerfrei die Absolutposition an jeder Position auf dem Maßstab bestimmt werden, ohne auf gespeicherte Daten zurück- 
greifen zu müssen oder eine Referenzfahrt durchzuführen. Zur Bewertung der Systemgenauigkeit wurde die inkrementelle Spur gegen das Referenzinterferometer verglichen (Abb. 18, unten). Über den gesamten Bewegungshub beträgt die Abweichung maximal $\pm 700 \mathrm{~nm}$. Dabei wurde auf die Rohdaten lediglich eine globale Offset- und Amplitudenkorrektur angewandt. Da durch die PRBS-Spur die absolute Position bekannt ist, kann das System zusätzlich kalibriert werden. Mit Hilfe einer Fehlertabelle lässt sich die Genauigkeit somit noch weiter verbessern [10].

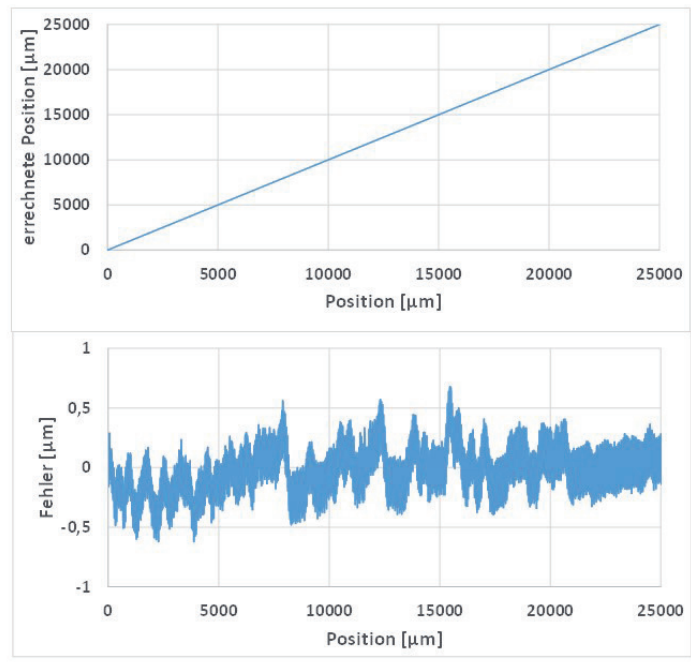

Abb. 18: Messergebnisse für miniaturisiertes Wegmesssystem

Aufgrund dieser hervorragenden Ergebnisse wurden die Maßstäbe und TMRLängensensoren unmittelbar in industrielle Anwendungen übertragen. Abb. 19 zeigt eine Fokussierachse für einen Mikroskop-Tisch der Projektpartner Märzhäuser. Mit diesem neuen Ansatz hat Sensitec mit den weiteren Projektpartnern die erreichbare Auflösung und Genauigkeit von magnetischen Längenmesssystemen in einen bisher unerreichten Bereich gebracht und bietet damit eine robuste Alternativ zu hochauflösenden optischen Längenmesssystemen.

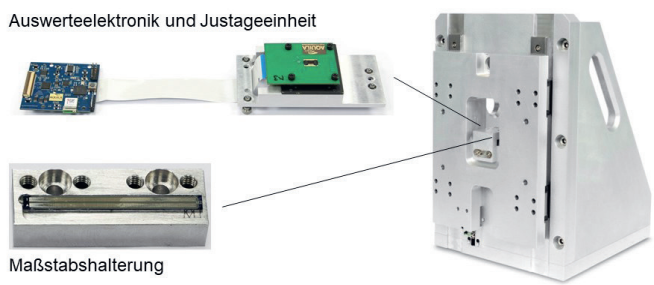

Abb. 18: Anwendungsbeispiel - Fokussierantrieb für Mikroskop (Bildquelle: Märzhäuser $\mathrm{GmbH} \&$ Co. KG)
5.3 Feldsensorik

Aufgrund der sehr kleinen Abmessungen und hohen Empfindlichkeit liegt es nahe, auch Feldsensoren auf TMR-Basis zu entwickeln. Abb. 20 zeigt eine typische Ausführung, wo der Feldsensor als Chip-Scale-Package umgesetzt wurde.

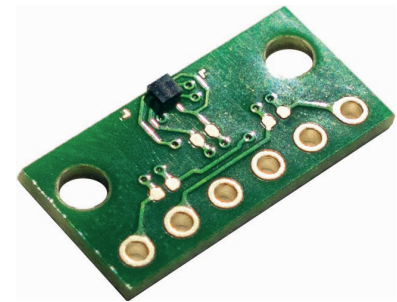

Abb. 20: Feldsensor auf TMR-Basis (Flip-Chip auf Leiterplatte)

Eine typische Anwendung ist die als Schaltsensor. Die Anordnung in Abb. 21 kann benutzt werden für Endlagen-Schalter in Linearzylindern auf elektrischer, pneumatischer oder hydraulischer Basis. Der Dipolmagnet wird auf der Schubstange angebracht und der TMR Feldsensor üblicherweise in das Zylindergehäuse integriert. Die hohe Empfindlichkeit des TMR-Sensors führt dazu, dass entweder der Abstand zwischen Magnet und Sensor vergrößert werden kann oder kleinere Magnete eingesetzt werden können.

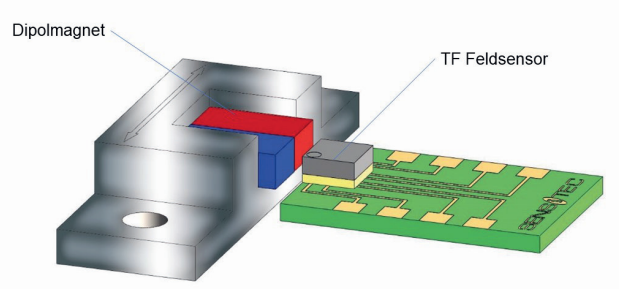

Abb. 21: Typische Messanordnung für TMRSchaltsensor

Eine weitere Anwendungsmöglichkeit für TMRFeldsensoren findet sich in Wegmesssystemen (Abb. 22). In Lineardirektantrieben ist es vorteilhaft die Statormagnete als Maßverkörperung zu nutzen, d .h. es bedarf keines separaten Maßstabs, wie es bei optischen Messsystemen erforderlich wäre. Um die Auswirkungen von geometrischen Fehlern der Magnetsegmente bzw. Teilungsfehlern zu unterdrücken, wird üblicherweise ein Array an Sensoren ausgeführt. Diese Anordnung ist besonders attraktiv in Handling-Applikationen, wo ausreichende Genauigkeit geboten wird trotz eines sehr einfachen und kompakten Aufbaus des Messsystems. 

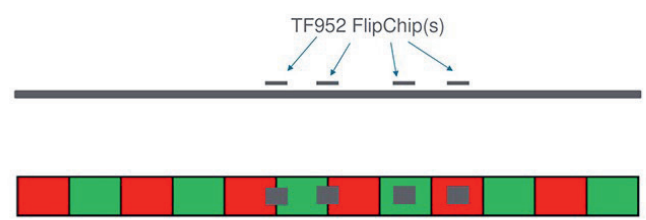

Abb. 22: Wegmesssystem mit TMR-Feldsensoren

\section{Zusammenfassung}

Steigende Anforderungen an Sensoren in der Antriebstechnik führen zu einem wachsenden Anteil an magnetischen Sensoren, insbesondere jener, die auf dem magnetoresistiven Effekt basieren. MR Sensoren sind ein gelungenes Beispiel für die Mikro-Nano-Integration. Aktuelle Neuentwicklungen bei Sensoren auf Basis des Tunnelmagnetoresistiven-Effekts öffnen neue Möglichkeiten für Antriebs- und Maschinenentwickler. Alle wichtigen Applikationen, um Bewegungen in der elektrischen Antriebstechnik zu erfassen, können mit TMRSensoren abgedeckt werden. Damit bilden TMR-Sensoren eine wichtige Ergänzung für die etablierten AMR- und GMR-Technologien.

Bei TMR-Sensoren verfügt eine neue Winkelsensorfamilie über eine höhere Winkelgenauigkeit und höhere Dynamik als bisherige TMR-Sensoren und auch Hall-Effekt Sensoren. Neue Fertigungstechnologien ermöglichen miniaturisierte Längenmesssysteme für komplett neue Anwendungen. Extrem kleine Feldsensoren sind sehr gut geeignet als EndlagenSchalter und ermöglichen deutlich kompaktere Konstruktionen.

TMR-Sensoren sind nicht nur für Winkel- und Längenmessaufgaben geeignet, sondern auch für Anwendungen im Bereich der mobilen $\mathrm{Na}$ vigation, des Verkehrsmanagements, der Strommessung und Zustandsüberwachung $[11,12,13]$.

\section{Literatur}

[1] Duret, C. et al, "TMR : a new frontier for magnetic sensing", Proc. of $11^{\text {th }}$ Symposium Magnetoresistive Sensors and Magnetic Systems, Wetzlar, 2011

[2] Paul,J., Lehndorff, R. \& Duret, C.; „Ressourceneffizienz am Beispiel magnetoresistiver Sensoren“, Mikrosystemtechnik Kongress 2011, Darmstadt

[3] Slatter, R., Doms, M., "Energy efficient magnetoresistive sensors for low-power and wireless applications", Proc. of Smart Systems Integration Conference, Vienna, 2014

[4] Slatter, R., Buss, R.; „Energieeffiziente, miniaturisierte magnetische Sensoren für energieautare Anwendungen", 8. GMM-Workshop Energieautarke Sensorik - EAS 2016, Renningen, 2016
[5] Doms, M. et al; "AMR vs. GMR vs. TMR - Eigenschaften, Unterschiede, Anwendungen", Proc. of 5. Mikrosystemkongress, Aachen, Germany, 2013

[6] Slatter, R.; „Neuartige magnetoresistive Sensoren für Winkel- und Längenmessaufgaben im Automobil“, Sensoren im Automobil IV, Expert Verlag, Renningen, 2011

[7] Slatter, R., von Manteuffel, G.; „Neue Entwicklungen bei magnetoresistiven Sensoren für Weg-, Winkel-, Strom- und Feldmessung in und um das Automobil“ in Automobil-Sensorik, Tille, T. (Hrsg.), Springer Vieweg, Wiesbaden, 2016

[8] Lindemann, R.; "KaLiPso - Wireless position measuring in linear guides using integrated TMR Sensors", Proc. of $14^{\text {th }}$ Symposium Magnetoresistive Sensors and Magnetic Systems, Wetzlar, 2017

[9] Slatter, R. \& Paul, J.; "A new approach to miniaturize high resolution linear position measurement systems", Proc. of $14^{\text {th }}$ Symposium Magnetoresistive Sensors and Magnetic Systems, Wetzlar, 2017

[10] Paul,J. et al; „High Resolution Magnetic Position Sensors for Precise Control of Production Tools“, Proc. of $15^{\text {th }}$ Symposium Magnetoresistive Sensors and Magnetic Systems, Wetzlar 2019

[11] Helwig, N.; "Modular Sensor Systems for real time Process Control and Smart Condition Monitoring using XMR sensor technology“, Proc. of $14^{\text {th }}$ Symposium Magnetoresistive Sensors and Magnetic Systems, Wetzlar, 2017

[12] Slatter, R., Holland, L. \& Abele, E.; „Magnetoresistive sensors for the condition monitoring of high-frequency spindles", Proc. of $7^{\text {th }}$ HPC CIRP Conference on High Performance Cutting, Chemnitz, 2016

[13] Traute, J., Casper, F. \& Paul, J.; „Robuste xMR-Sensoren für die Zustandsüberwachung", 20. GMA/ITG Fachtagung Sensoren \& Messsysteme, Nürnberg, 2019 